« Les accidents de la vie. » Maladie, traumatisme et création chez Blaise Pascal ${ }^{1}$

Tony Gheeraert Université de Rouen / CEREDI

Dans l'histoire des idées, certaines thèses ont la vie dure et résistent à tous les bouleversements des paradigmes épistémologiques. L'hypothèse qu'un lien obscur s'établirait entre talent créateur et disposition morbide est de celles-là ; on peut en retracer la généalogie, qui remonte à la théorie médicale de l'humeur noire : le Problème $X X X$, attribué à Aristote, postulait déjà que le génie dans tous les domaines procédait d'une complexion mélancolique ${ }^{2}$. La ruine de l'ancienne médecine humorale ne suffit pas à entraîner celle de la bile noire, qui offrait un modèle étiologique du génie trop commode et trop séduisant pour qu'on y renonce facilement : folie et créativité restent, au moins dans l'imaginaire, unies par un lien aussi mystérieux qu'essentiel. Les ouvrages critiques cherchant dans la folie supposée d'Artaud, de Nerval, voire de Maupassant, le secret de leur œuvre, trop nombreux pour qu'on les recense ici, attestent de la fécondité, sous des formes adaptées à l'époque contemporaine, de cet ancien postulat.

\title{
Un « cas » Pascal ?
}

Blaise Pascal aussi, sans avoir pourtant jamais été atteint de démence, n'a cessé de susciter l'intérêt de commentateurs avides de découvrir dans sa maladie, voire dans une forme de névrose causée peut-être par une pratique religieuse atypique, la source et l'explication de son œuvre protéiforme. Se pouvait-il après tout qu'un "génie » aussi divers ait été «normal »? La question n'est pas neuve, et les contributions sur le sujet sont fort nombreuses. Des lecteurs ordinaires aux plus savants médecins, le « cas» Pascal intéresse, exalte, fascine, obsède. Paralysie, accident vasculaire cérébral, et

1 La présente étude a d'abord été prononcée le 19 mars 2010 à l'université de Rouen, dans le cadre du colloque Traumatismes et pensées, organisé par le laboratoire Psy-NCA, Équipe Traumatismes Individuels et Familiaux.

2 Aristote, Problèmes, XXX, 1, 953a10-14. 
jusqu'à la rage de dents, rien n'aurait été épargné au grand homme, comme si les forces de la nature s'étaient liguées à la fois pour l'inciter et pour l'empêcher de mener à bien les tâches prométhéennes qu'il avait entreprises, entre autres cette gigantesque apologie dont on ne possède que des fragments. Admirateurs et détracteurs, scientifiques et littéraires, professionnels et amateurs ont étudié, interprété, commenté à perte de vue les «symptômes» supposés de Blaise Pascal. Récemment encore, un article de Psychanalyse Magazine proposait un diagnostic complet de notre analysant malgré lui, et concluait à un syndrome «maniaco-dépressif » directement lié à une succession de traumas subis par le malheureux Blaise dès son enfance :

Blaise, enfant anxieux, dépressif, est, en bas âge, pris de convulsions. Il souffre en outre d'une peur panique de l'eau, et la naissance de sa petite sœur Jacqueline n'arrange rien. Blaise, accroché à l'objet d'amour maternel, connaît sa première grande déception amoureuse et cette blessure narcissique le fait sombrer dans la mélancolie. S'étant fantasmé le préféré de sa mère depuis sa maladie, il ressent comme une trahison l'arrivée de sa puînée. Il se vit dès lors abandonné, subit du même coup le sevrage du sein, éprouvant là une perte douloureuse. Blaise développe beaucoup d'hostilité envers sa mère $[\ldots] .^{3}$

La suite de l'article continue à énumérer les catastrophes, réelles ou perçues comme telles, qui se seraient accumulées sur la tête, les jambes, et tout l'organisme du pauvre enfant malingre. Si l'on ne voit pas bien quelles conclusions tirer du «sevrage du sein », trauma que Pascal partage avec l'ensemble de l'humanité depuis qu'elle existe, on comprend bien l'orientation générale des propos du biographe amateur: il s'agit pour lui de dresser un inventaire de symptômes susceptibles de rendre compte de l'œuvre ultérieure de Pascal. Un tel diagnostic, lointainement inspiré par le texte de Freud sur un souvenir d'enfance de Goethe ${ }^{4}$, peut prêter à sourire ; de tels raccourcis ne sont pourtant pas seulement le fait de journalistes à sensation pressés ; à travers les siècles, les lecteurs n'ont cessé de se pencher sur l'origine des maux dont souffrit l'auteur des Pensées: Pascal travaillé par le bouillonnement de ses humeurs au XVII ${ }^{\mathrm{e}}$

3 Luc Carlier, «Blaise Pascal, un maniaco-dépressif?», in Signe et Sens, http://www.psychanalysemagazine.com/psychobiographie-blaise-pascal-un-maniaco-depressif.htm

4 «Un souvenir d'enfance dans Fiction et Vérité de Goethe» [1917], trad. M. Bonaparte [1933]. Luc Carlier s'arrête tout particulièrement ici sur le fantasme de préférence maternelle et la jalousie envers la cadette : on se souvient que Freud avait montré, dans «Un souvenir d'enfance de Goethe », que ces éléments avaient été des ingrédients essentiels dans la genèse du romantique allemand; aux yeux de Luc Carlier, la supériorité éclatante du classique français semble bien procéder de souffrances enfantines du même type que celle du romantique allemand. 
siècle, Pascal nerveux au XIX ${ }^{\mathrm{e}}$, Pascal névrosé, voire «maniaco-dépressif » au $\mathrm{XX}^{\mathrm{e}}$ et $\mathrm{XXI}^{\mathrm{e}}$, c'est toute la panoplie des grandes affections psychologiques qui a servi à qualifier ses troubles. Les appellations changent, le mythe reste. Car l'intérêt d'une telle déferlante exégétique, avant même d'évaluer son degré de véracité, réside dans ce que révèlent les présupposés, implicites ou explicites, d'une pareille démarche. Il est trop tard, maintenant, pour espérer encore pouvoir soigner Pascal, et il est clair qu'on ne s'interrogerait pas sur les indispositions multiples de l'auteur des Pensées et de l'inventeur du vide s'il n'était l'un et l'autre. Le « cas Pascal » n'a suscité un semblable intérêt que parce qu'on espérait trouver dans l'examen clinique du grand homme une corrélation entre son état de santé déplorable et les étonnantes productions de son esprit. Les lecteurs les moins bien intentionnés attribuent ses travaux au dérangement de son cerveau. D'autres, lointains descendants d'Aristote et Galien, considèrent au contraire que c'est son talent supérieur qui s'ancre dans des déterminations d'ordre physiologique : pour ces derniers, Blaise, enfant pas tout à fait comme les autres, et dont la vie fut traversée par de lourdes épreuves, aurait en quelque sorte été prédestiné (osons le mot) tant à la souffrance physique et morale qu'à l'absolu d'un génie inégalable.

Ce sont ainsi deux préjugés, l'un favorable et l'autre défavorable, mais qui constituent comme l'avers et le revers d'une même médaille, que l'on se propose ici d'interroger, non afin de diagnostiquer les maladies et les traumatismes dont a souffert Pascal, mais pour comprendre les raisons de l'insistance mise par les commentateurs à surinterpréter, à exagérer, voire à fabuler les symptômes morbides de l'auteur des Pensées. Afin d'éviter la pétition de principe consistant à prêter d'abord à Pascal des maux plus ou moins bien identifiés, l'enquête commencera par mesurer, autant que possible, l'intensité et la nature des troubles dont il souffrait; elle se poursuivra ensuite par l'examen dans son œuvre du thème morbide, dont l'importance ne doit pas nécessairement être référée à une expérience personnelle, mais plutôt à la pensée théologique qui sous-tend son écriture. Ce n'est qu'une fois circonscrite la part de la légende et celle de la pensée augustinienne que la question d'un lien éventuel entre maladie, traumatisme et création pourra être de nouveau posée.

\section{Deux ou trois choses qu'on sait de lui}


«Assurons-nous bien du fait, avant que de nous inquiéter de la cause », prescrivait Fontenelle. Avant de mettre en place de lourdes procédures herméneutiques, encore faut-il vérifier la solidité des sources. De quoi souffrait Pascal ? Quels maux étaient les siens, quels traumatismes a-t-il vécus? Notre savoir sur le sujet repose pour l'essentiel sur deux textes. Le plus important est la Vie de Monsieur Pascal établie peu après la mort de Blaise par sa sœur Gilberte, et souvent rééditée ${ }^{5}$; le second est un mémoire rédigé plus tard par la fille de cette dernière, Marguerite, celle-là même qui fut guérie d'une fistule lacrymale en 1656, après avoir touché une relique de la SainteÉpine'. Or, la fiabilité de ces deux récits, dans lesquels les biographes de Pascal n'ont cessé de puiser à pleines mains, est loin d'être assurée. Jean Mesnard note ainsi, dans la minutieuse étude qu'il consacre à la vie écrite par Gilberte, que le «degré de confiance qu'il convient d'accorder à ce document fondamental [...] n'a jamais encore été qu'effleuré ».

[...] Publiée quelque cent fois, citée, paraphrasée dans mille ouvrages, cette Vie semblerait ne devoir réserver aucune surprise. Or, à celui qui l'examine de près, elle offre toutes sortes d'énigmes. [...] La valeur historique de ce grand témoignage a fait l'objet d'appréciations contradictoires, mais non d'études critiques. [...] Au fond, la critique n'a jamais mis profondément en doute la véracité de Gilberte. ${ }^{7}$

De fait, il est difficile d'évaluer la pertinence de ce témoignage direct: Gilberte, qui partagea surtout l'enfance et les dernières années de son frère, fut moins intime avec lui pendant la période où il fut particulièrement actif. La question des sources pose également problème : la vérification des propos prêtés à Pascal est impossible, et on en est réduit à des conjectures ; on suppose que la biographe s'appuie, outre ses souvenirs, sur un livre de raison, des lettres, ou encore un recueil de sentences, dont on connaît l'existence par ailleurs mais qui a été depuis perdu. Sa relation comporte, en tout état de cause, quelques fautes manifestes : l'abandon des sciences par Pascal est situé par sa sœur en 1646, date manifestement trop précoce, antérieure même aux grandes expériences sur le vide. Jean Mesnard, tout en estimant que «l'essentiel nous a bien été

5 Nous nous référerons dans cette conférence aux Euvres complètes de Pascal, annotées et éditées par Jean Mesnard chez Desclée de Brouwer (4 tomes parus à ce jour depuis 1964). La Vie écrite par Gilberte est reproduite au tome 1, p. 571-602.

6 «Mémoire sur Pascal et sa famille », ibid., p. 1090-1105.

$7 \quad$ Ibid., t. I, p. 539 et 565. 
livré », note que, quand elle est possible, la confrontation avec d'autres sources confirme les dires de Gilberte; il reconnaît néanmoins chez elle une propension à « l'idéalisation », voire aux « déformations ». Ainsi, la sœur du savant s'abstient de tout commentaire sur les Provinciales, et l'omission surprend. A-t-elle pensé que les Petites Lettres, polémiques et hostiles à Rome, jetaient une ombre au tableau de la sainteté de son frère ? Par ailleurs, Philippe Sellier, dans un article qui fit date, montra que la Vie de Monsieur Pascal, sans être en rien fictionnelle ni mensongère, ne peut être lue comme un simple témoignage à l'état brut: Gilberte obéit à la pression d'un genre littéraire et hagiographique, celui de la «légende ${ }^{8} »$ : Gilberte agence les éléments biographiques qu'elle relate de façon à se conformer aux règles habituelles d'une vie de saint, tel que le genre était pratiqué depuis le Moyen-Âge. La forme ici fait sens : elle tend à imposer l'idée que Pascal bénéficia de la grâce efficace et du don de persévérance, et peut ainsi à juste titre être compté parmi les grandes figures du christianisme. De telles analyses ne suffisent pas à révoquer en doute le témoignage de Gilberte : les amis de Port-Royal étaient trop intransigeants avec l'exigence absolue de vérité pour qu'on les soupçonne d'une falsification délibérée destinée à glorifier l'un des leurs99. Il n'en reste pas moins que de telles pages, tout entières conditionnées par des impératifs d'ordre religieux ou littéraire, ne sauraient être considérées comme un document neutre du point de vue historiographique. Il serait injuste de conclure à la mystification, mais il n'en reste pas moins que la Vie de Monsieur Pascal est une œuvre trop concertée, élaborée avec trop de science, conçue en fonction de finalités et de considérations formelles trop particulières et trop contraignantes pour qu'on la traite comme un exposé brut et objectif, dans lequel Gilberte se serait contentée de transcrire ses souvenirs au fil de la plume.

S'il convient de faire preuve de prudence lorsqu'on aborde la biographie laissée par Gilberte, une circonspection bien plus grande encore est de mise dès qu'on regarde

8 Philippe Sellier, «Pour une poétique de la légende, la Vie de M. Pascal », Chroniques de Port-Royal, 31, 1982, p. 51-56. Article repris dans Port-Royal et la littérature. Pascal, deuxième édition, augmentée de douze études, Honoré Champion, «Champion Classiques - Essais », 2010, p. 35-61. Voir aussi, dans le même recueil, «La version amplifiée de La Vie de Monsieur Pascal », p. 63-100.

9 Jehanne d'Orliac avait autrefois tenté, sans emporter la conviction, d'éreinter l'auteur des Pensées en accusant Gilberte d'affabulation (Le Cour humain, inhumain, surhumain de Blaise Pascal, Baudinière, 1931). 
le mémoire de Marguerite ${ }^{10}$. Témoignage de seconde ou de troisième main, rédigé par une descendante qui a peu connu Pascal, ce récit est aussi riche en anecdotes que le premier en est dépourvu. Les biographes ultérieurs n'emprunteront que plus volontiers à ces deux sources si complémentaires. Pourtant, ces deux textes ne sont pas à mettre sur le même plan : si la Vie de Monsieur Pascal fut rédigée par une intime de Blaise très peu de temps après la mort prématurée du savant, le Mémoire fut composé à une date bien plus tardive, entre 1723 et 1732, alors que Marguerite était elle-même fort âgée. Son opuscule n'offre par ailleurs ni la même rigueur ni la même qualité de style que celui de sa sœur; il arrive ainsi à la narratrice, qui affectionne les détails au détriment d'une vue d'ensemble, de se contredire gravement d'un passage à l'autre. Le goût de l'anecdote se double chez elle d'une attirance suspecte pour le merveilleux : on a ainsi retrouvé, pieusement conservé dans ses papiers, un mémoire port-royaliste anonyme relatant comment un fossoyeur découvrit un jour le corps et les vêtements de la Mère Angélique, miraculeusement épargnés par le pourrissement ${ }^{11}$. De fait, c'est dans le Mémoire de Marguerite, et sous les auspices du surnaturel, que prend naissance la légende de Blaise, et qu'est relaté le premier traumatisme auquel il aurait été confronté.

La nièce de Pascal rapporte en effet que, dans sa prime enfance, le petit Blaise aurait souffert de troubles du comportement (phobie de l'eau, crises de colère lorsqu'il voyait ses parents ensemble) et serait «tombé en chartre », c'est-à-dire qu'il aurait souffert d'un amaigrissement et d'une perte de vitalité. Toujours d'après Marguerite, l'entourage de l'enfant se serait avisé qu'un tel mal n'avait pu être provoqué que par une cause diabolique ; la famille jeta sa vindicte sur une pauvre vieille à qui on faisait jusque-là la charité, et qu'on eut tôt fait d'accuser de sorcellerie. Le père de Blaise, Étienne, aurait obtenu d'elle des aveux, à force de menaces, et l'aurait contrainte à annuler son sort; elle aurait accepté, après diverses tractations, de transporter le sortilège sur un chat. Loin de guérir, le garçonnet serait tombé dans une sorte de coma : « il avait les apparences de la mort ». La sorcière, interrogée, supplia Étienne d'attendre minuit, et lui assura que l'enfant reviendrait ensuite à la vie, ce qui arriva. Pour comble

\footnotetext{
10 Blaise Pascal, Euvres complètes, op. cit., t. I, p. 1091-1055.

11 Ibid., p. 1147-1148.
} 
de bonheur, une fois remis, l'enfant ne tarda pas à guérir également de sa phobie aquatique, et accepta même de bon gré d'assister aux embrassades de ses parents. Marguerite termine ce conte bleu en concluant que «depuis, il n'eut plus jamais aucun mal ».

La seule chose qui surprenne vraiment dans ce récit, c'est le sérieux dans lequel on l'a tenu et on le tient encore. Peut-on seulement démêler le vrai du faux dans cette histoire ? À quelle maladie renvoie par exemple l'expression «tomber en chartre » ? À la phtisie, comme le pense Furetière ? Les auteurs modernes donnent en effet le mot comme synonyme «d'atrophie mésentérique » qui survient dans la tuberculose, mais, si tel avait été le cas, le malade n'aurait pas survécu. Au rachitisme ? Les médecins d'autrefois confondaient volontiers l'atrophie mésentérique, dont le pronostic est fatal mais qui touche rarement de si jeunes enfants, et le «carreau » ou rachitisme, dont les symptômes sont proches, et qui se traduit par des atteintes hépatiques. Certains éléments confirmeraient ce diagnostic, comme les convulsions et les atteintes musculaires ; l'évolution habituelle du rachitisme est spontanément favorable, ce qui expliquerait la guérison, mais cette affection laisse des séquelles, notamment osseuses, qui retardent en particulier la fermeture des fontanelles ; or, précisément, on sait grâce aux conclusions de l'autopsie réalisée après son décès que les os du crâne de Pascal étaient déformés ${ }^{12}$.

Il n'en reste pas moins qu'on en reste ici à d'invérifiables conjectures, et que bien des points invitent à la plus grande réserve. Les commentateurs s'étonnent de la crédulité que Marguerite prête à Étienne : cet esprit éclairé, reçu chez les plus grands savants de son siècle, où il introduira plus tard son fils, était a priori peu enclin à prêter foi à des histoires de loups garous. André Le Gall suppose, pour expliquer le comportement superstitieux d'Étienne, que sa grande douleur de père avait dû affaiblir sa capacité à raisonner, et qu'il était prêt à tout pour sauver son fils, même à croire les contradictions puériles d'une pauvre vieille aux abois. Il ajoute que ce témoignage vaut

12 Ibid., p. 646 : «Le crâne se trouva sans aucune suture que la [lambdoïde ou la sagittale ?], ce qui apparemment avait causé les grands maux de tête auxquels il avait été sujet pendant sa vie. Il est vrai qu'il avait eu autrefois la suture qu'on appelle fontale ; mais ayant demeuré ouverte fort longtemps [...], et n'ayant pu se refermer, il s'était formé un calus qui l'avait entièrement couverte, et qui était si considérable qu'on la sentait aisément au doigt. Pour la suture coronale, il n'y en avait aucun vestige ». 
comme document sur l'état des mentalités en Auvergne dans le premier tiers du XVII siècle ${ }^{13}$.

Conclusion prudente, à laquelle on ne saurait toutefois souscrire sans précaution. Bien des éléments nous inviteraient en fait à formuler un jugement plus radical. D'abord, la source elle-même est sujette à caution: Marguerite ne relate pas directement l'anecdote telle qu'elle aurait pu l'apprendre de sa mère, mais elle démarque presque mot à mot un de ces mémoires anonymes qu'elle recueillait ${ }^{14}$. De plus, les invraisemblances fourmillent : mettons que, tenaillé par la douleur, Étienne ait cru à une possession démoniaque, et qu'il ait accepté les négociations douteuses avec la vieille femme, proposant deux chats après avoir refusé un enfant et offert un cheval encore que la scène tienne plus de comices agricoles burlesques que d'une séance de désenvoûtement. Mais que Blaise, guéri de sa crise de sa «chartre », ait été du même coup soigné de ses phobies, voilà qui est fort douteux, d'autant que c'est Marguerite qui introduit explicitement le lien entre peurs et convulsions, dont sa source ne dit rien. Ces dernières, on le sait aujourd'hui, ont le plus souvent une cause biologique, même si l'on rapporte des cas de convulsions provoquées par la hantise de l'eau dans des affections comme la rage. La guérison simultanée du rachitisme et des angoisses œdipiennes qui en seraient à l'origine est toutefois pour le moins étonnante, et ne plaide pas, en tout cas, en faveur de la solidité du témoignage.

Jean Mesnard, qui met en avant les nombreuses inconnues du texte, invite à considérer le mémoire original avec, au mieux, la plus grande réserve, et doute que les paroles prêtées à Gilberte aient été «bien comprises » par sa fille. Il recommande « la prudence» à «quiconque voudrait fonder sur cette anecdote une étude de la psychologie de Pascal. $»^{15}$

Pour apprécier à sa juste mesure la portée de cette historiette, il convient de rappeler que son auteur est elle-même une miraculée. Le miracle est un traumatisme : on ne sort pas indemne d'une expérience comme celle-là, surtout lorsqu'elle est vécue

\footnotetext{
13 André Le Gall, Pascal, Flammarion, « Grandes biographies », 2000, p. 51.

14 Mémoire reproduit par Jean Mesnard dans Blaise Pascal, Euvres complètes, op. cit., t. I, p. 507-508.

15 Ibid., p. 504.
} 
dans l'enfance : en quelques heures, par l'opération d'une relique de la Sainte-Épine, Marguerite, malade incurable, a recouvré la santé. On conçoit qu'une telle guérison ait dû rendre la nièce de Pascal particulièrement réceptive aux merveilles du miracle, mais on ne saurait exclure qu'elle ait développé par la suite une propension à la crédulité. Si son récit peut constituer un document sur l'histoire des mentalités, il concerne sans doute moins le climat intellectuel de l'Auvergne des années 1620 que celui du jansénisme à l'époque des convulsionnaires de Saint-Médard.

Plus inquiétant encore : c'est au seul témoignage de Marguerite que l'on doit la plupart des descriptions des troubles vécus par Blaise dans sa vie adulte. Ainsi, elle est la seule à évoquer la paralysie « de la ceinture jusques en bas » qui, d'après elle, frappa son oncle vers la fin de la période rouennaise (1647). On a beaucoup glosé sur cette affection, dont on dit qu'elle peut expliquer en partie le départ de Blaise pour Paris. Gilberte, dans sa Vie, évoque de son côté une première grave attaque à l'abdomen et à la tête. La mention de la paralysie par Marguerite reste isolée : dans la lettre de Jacqueline à Gilberte datée du 25 septembre 1647, au plus fort de la crise, nous voyons Blaise aphone et migraineux, mais il n'est fait aucune mention de cette impossibilité de marcher; bien au contraire, l'épistolière nous apprend que son frère se rend «à l'église », tandis que Descartes, médecin de fortune rencontré à ce moment-là, conseille au malade de garder le lit tant qu'il n'en est pas las. Les critiques concluent généralement à une paralysie intermittente, mais on peut tout aussi bien purement et simplement révoquer en doute les allégations de Marguerite.

Pascal fut-il si malade qu'on l'a dit ? Souffrit-il tous les troubles physiques et les chocs psychologiques qu'on lui a prêtés ? La notice rédigée par Régine Pouzet pour le Dictionnaire de Port-Royal n'accorde qu'une place minime à la maladie, et précise que, jusqu'à ses dernières années, Blaise fut relativement épargné par la douleur ${ }^{16}$. Certes, on ne prétendra pas que Pascal fut un bien portant qui s'ignorait: il fut frappé dès sa jeunesse par de redoutables maux de ventre et de tête, connut quelques rémissions

16 Réginet Pouzet écrit, à propos de la crise de 1660 : «Comme par le passé, l'intestin et le cerveau sont le plus touchés, mais aucune allusion n'est faite à de grandes souffrances. L'essentiel consiste en une perte générale des forces, rendant impossible tout travail, surtout intellectuel » (Dictionnaire de Port-Royal, éd. J. Lesaulnier et Antony McKenna, Paris, Honoré Champion, 2004, p. 785). 
malgré les traitements que les médecins lui infligeaient, et fut emporté précocement, sans doute à la suite d'une rupture d'anévrisme, comme en témoigne le « sang caillé » retrouvé dans son crâne après sa $\operatorname{mort}^{17}$; ces atteintes physiques furent moins spectaculaires que beaucoup, pour des raisons différentes, se sont plu à le penser. Le reste n'est qu'incertitudes. La vraie question consisterait à se demander à quoi attribuer cette propension, assez générale chez les biographes jusqu'à une époque récente, à exagérer les maux (au demeurant réels) qu'eut à souffrir l'auteur des Pensées.

\section{La maladie, thème augustinien}

La première explication réside dans la place occupée par la maladie dans le système religieux professé par les amis de Pascal. Augustin accorde en effet une place centrale au péché originel, qui constitue de ce point de vue un traumatisme au sens qu'on donne d'habitude à ce mot, c'est-à-dire de dommage causé à la structure et au fonctionnement du corps et de l'âme. En désobéissant à Dieu, Adam a renoncé à l'état d'innocence et de perfection qui était le sien, pour tomber dans un état de misère, d'aveuglement, de maladie, de corruption entraînant la mort, tant corporelle que spirituelle. Le premier homme a été créé «sans tache, juste [...], pur, saint, parfait » et sa nature est désormais, par sa faute, «souillée, abominable, détestable aux yeux de Dieu $^{18} \gg$. La métaphore de la maladie contagieuse sert à Pascal à faire comprendre, autant que cela est possible, la transmission du péché originel, que le père de l'humanité a passé mystérieusement à tous ses descendants : «Adam [...] a corrompu et infecté toute la masse des hommes ${ }^{19}$ ». Il précise dans le deuxième écrit sur la grâce : « $\mathrm{Ce}$ péché ayant passé d'Adam dans sa postérité, qui fut corrompue en lui comme un fruit sortant d'une mauvaise semence ${ }^{20} »$. La culpabilité des descendants d'Adam est ainsi

\footnotetext{
17 «Mais ce qu'on remarqua de plus considérable, et à quoi on attribua particulièrement sa mort et les derniers accidents qui l'accompagnèrent, fut qu'il y avait au-dedans du crâne, vis-à-vis les ventricules du cerveau, deux impressions, comme du doigt dans de la cire, qui étaient pleines d'un sang caillé et corrompu et qui avait commencé de gangréner la dure-mère », in Pascal, Euvres complètes, op. cit., t. I, p. 646.

18 Blaise Pascal, Écrits sur la grâce, ibid., t. III, p. 787.

19 Ibid., p. 787-788.

20 Ibid., p. 794.
} 
présentée non comme non juridique, mais biologique : le péché est comparable à une affection transmissible (« infection»), ou à une gangrène cancéreuse (« corruption »).

C'est dans cette perspective augustinienne qu'il convient de comprendre la Prière sur le bon usage des maladies, rédigée par Pascal sans doute à la fin de l'année 1660²1. Le titre de cet opuscule est dû à Gilberte ${ }^{22}$, qui fut la première à mentionner son existence et à suggérer une date de composition. Après une édition pirate parue en 1666, les Périer s'emploient à en donner un texte correct qui voit le jour avec l'édition de Port-Royal des Pensées, au début de l'année 1670. Comme souvent chez Pascal, la méditation s'organise autour d'un de ces «renversements du pour au contre » qui constituent le principe même de son écriture : aux yeux du monde, la santé paraît désirable, mais en fait, dans la mesure où elle donne l'occasion de goûter trop facilement aux fausses joies du siècle, elle est un piège dangereux.

Vous m'aviez donné la santé pour vous servir, et j'en ai fait un usage tout profane. Vous m'envoyez maintenant la maladie pour me corriger ; ne permettez pas que $\mathrm{j}$ 'en use pour vous irriter par mon impatience. J'ai mal usé de ma santé, et vous m'en avez justement puni. Ne souffrez pas que j'use mal de votre punition. Et puisque la corruption de ma nature est telle qu'elle me rend vos faveurs pernicieuses, faites, ô mon Dieu ! que votre grâce toute puissante me rende vos châtiments salutaires. Si j'ai eu le cœur plein de l'affection du monde pendant qu'il a eu quelque vigueur, anéantissez cette vigueur pour mon salut ; et rendez-moi incapable de jouir du monde, soit par faiblesse de corps, soit par zèle de charité, pour ne jouir que de vous seul. ${ }^{23}$

Il est presque impossible de se sauver lorsqu'on jouit d'une parfaite santé : la tentation des plaisirs est trop forte pour pouvoir résister aux sortilèges des plaisirs d'ici-bas. La maladie seule peut, sous certaines conditions, être un bien : Dieu ne permet que nous soyons touchés par elle qu'en vue de sauver ceux qu'il a prédestinés. Dans la mesure où le mal donne à voir dans le corps l'état réel de l'âme gangrenée par le péché, il peut nous purifier de nos attachements aux choses périssables et ainsi être une voie du salut. Encore faut-il en faire, un «bon usage » : ceux pour qui la maladie est occasion de révolte et de blasphème contre la volonté insondable du Tout-Puissant montrent par là

21 Voir sur ce point l'introduction de Jean Mesnard à son édition de la Prière (ibid., t. IV, p. 978).

22 «On ne peut mieux connaître les dispositions particulières dans lesquelles il souffrait toutes ses nouvelles incommodités des quatre dernières années de sa vie [1659-1662] que par cette prière admirable que nous avons apprise de lui et qu'il fit en ce temps-là pour demander à Dieu le bon usage des maladies », ibid., t. I, p. 624.

23 Ibid., p. 998-999. 
l'endurcissement irrémédiable de leur cœur. En revanche, ceux qui «se soumettent à la douleur et à la souffrance ${ }^{24} \gg$ accueillent la maladie comme une grâce ; ils comprennent que Dieu l'accorde pour priver les pénitents de la jouissance des faux biens terrestres, et ainsi les attirer vers la vraie félicité. Comme l'écrit Laurent Thirouin : «la maladie est finalement indifférente. Elle aura la valeur que nous pourrons lui donner ${ }^{25} »$.

La maladie est par ailleurs un salutaire rappel des limites de la condition humaine : bien portants, nous vivons comme si nous étions immortels et nous nous « divertissons » sans penser à l'essentiel ; malades, nous sommes conduits à rentrer en nous-mêmes. La maladie nous invite ainsi à nous conformer au Christ en croix, et à unir notre volonté à la sienne, de sorte que nos maux, à la fois figures et châtiments du mal intérieur qu'est le péché, puissent permettre, avec l'aide de Dieu, de nous sauver. Il ressort de ce poème difficile que la maladie est non seulement une épreuve, mais aussi, à bien y regarder, une des plus hautes faveurs que Dieu puisse accorder à ses élus. Felix morbus que ce mal dont le Tout-Puissant se sert pour nous procurer un bien plus grand que la santé du corps, provisoire et illusoire : «par une radicale inversion, la maladie est devenue remède », note Laurent Thirouin ${ }^{26}$.

Il n'est pas aisé de tirer des conséquences éventuelles d'un tel texte sur les liens entre maladie et création chez Pascal. Certes, la Prière apparaît comme le produit d'une sublimation : la maladie est « convertie » non seulement en vertu, mais en chef-d'œuvre esthétique, et l'art rachète ainsi, d'une certaine manière, les brûlures de la souffrance. Force est de constater toutefois que Pascal ne théorise pas cette transmutation littéraire de la maladie. Par ailleurs, si l'ancrage autobiographique de ce texte, resté inédit à sa mort, est incontestable, il ne saurait suffire à épuiser la portée d'un opuscule qui excède les limites du «je » souffrant en relation intime avec son Créateur. Jean-Paul Amman,

24 La formule est de Henry Phillips («Pascal et les Pensées; négligence de la douleur, douleur de la négligence », Ull Critici, ${ }^{\circ}$ 9-10, 2005, p. 231-243).

25 Laurent Thirouin, «La santé du malheur. Santé et maladie dans la Prière pour demander à Dieu le bon usage des maladies », in Pascal, auteur spirituel, textes réunis par Dominique Descotes, Paris, Editions Honoré Champion, «Colloques, congrès et conférences sur le classicisme », 2006, p. 289.

26 Ibid., p. 295. L'auteur insiste sur l'opposition augustinienne de l'usage et de la jouissance « à l'œuvre dans tout le texte », et «clef ultime de toutes les attitudes qu'on peut avoir à l'égard de la santé et de la maladie». 
dans l'étude qu'il consacre à cette oraison ${ }^{27}$, montre, sans nier la dimension personnelle d'une pareille prière, qu'elle correspond à une volonté du méditant de s'unir en pensée avec la communauté des croyants, dont il était isolé du fait de sa maladie. Aussi ces pages, quelle qu'en soit la dimension individuelle irréductible, sont-elles destinées à l'ensemble des chrétiens qui, en vertu du dogme de la communion des saints, sont invités à reprendre à leur compte la méditation proposée par l'auteur : celui-ci « espère [par la grâce de Dieu] faire [de ses lecteurs] une portion [des élus] ». Prière littéraire, destinée à être prononcée par d'autres que celui qui l'a écrite, adressée à tous les fidèles et particulièrement à ceux qui souffrent dans leur chair, écrite de plus alors que Pascal ressent un apaisement de ses maux après la grave crise de 1659, elle est de ce fait pourvue d'une dimension universelle qui rend difficile toute conclusion se bornant à chercher une corrélation entre la maladie et l'œuvre ${ }^{28}$. Texte éminemment subjectif, la Prière ne saurait toutefois se réduire à un exercice solipsiste, comme le confirme le soin pris par l'auteur d'éviter toute référence aux affections précises dont il souffre.

Le socle théologique est trop important, et rend compte de façon trop cohérente des références morbides de l'œuvre pascalienne pour qu'on ignore son importance capitale dans la genèse des textes. La prégnance de cette thématique permet aussi de prendre la mesure de l'orientation qui gouverne les récits de Gilberte et de Marguerite, soucieuses de montrer la conformité de la vie de leur parent avec la doctrine qui leur était chère. Les catégories de l'augustinisme sont mobilisées à chaque page : ainsi, pour montrer que le jeune prodige fuyait les plaisirs des sens, Gilberte recourt à la distinction, typiquement augustinienne, de l'usage et de la jouissance. Comme le préconise Augustin, Pascal en effet use des créatures sans en jouir :

Il n'était pas possible qu'il n'usât de ses sens; mais quand il était obligé par nécessité de leur donner quelque plaisir, il avait une adresse merveilleuse pour en détourner l'esprit afin qu'il n'y prît point de part. Nous ne lui avons jamais ouï louer en parlant les viandes qu'on lui servait. ${ }^{29}$

27 Jean-Paul Amman, «La 'Prière' de Pascal 'pour demander à Dieu le bon usage des maladies' », Revue de l'histoire des religions, 2000, 217, p. 381-396.

28 En fondant l'analyse sur des critères étroitement biographiques, la critique a longtemps daté par erreur cette prière de 1659, date de la terrible crise qui frappa Pascal. Voir Jean Mesnard, Pascal, Paris, Desclée de Brouwer, «Les écrivains devant Dieu », 1965, p. 18-19.

29 Vie de Monsieur Pascal, in Euvres complètes, op. cit., t. I, p. 613. 
Loin de tirer la moindre satisfaction du corps et des sens, il s'inflige au contraire des blessures pour mieux châtier la chair coupable :

L'esprit de la mortification [...] lui inspira d'avoir une ceinture de fer pleine de pointes et de la mettre à nu sur sa chair toutes les fois qu'on le viendrait avertir que des messieurs le demanderaient. Il le fit, et lorsqu'il s'élevait en lui quelque esprit de vanité, ou lorsqu'il se sentait touché du plaisir de la conversation, il se donnait des coups de coude pour redoubler la violence de ces piqûres, et se faire ensuite ressouvenir de son devoir. ${ }^{30}$

Surtout, Gilberte s'attarde à mettre en évidence avec quelle vertu son frère supporta ses maux physiques, comme une épreuve que Dieu lui infligea pour purifier son âme. Jamais, même dans son agonie, il ne fut tenté d'incriminer le Ciel : sa souffrance fut au contraire l'instrument de sa Rédemption. Au moment de sa dernière maladie, tout son entourage est inquiet, car il semble que Pascal va décéder sans avoir le temps de recevoir les sacrements : "nous avions cet extrême déplaisir, avec tous les autres, de le voir mourir sans communier, après avoir demandé si souvent cette grâce avec tant d'insistance ${ }^{31} \gg$; heureusement, il bénéficie d'un répit qui lui permet de les recevoir en pleine lucidité. En montrant comment Pascal a supporté avec constance les maux envoyés par le Ciel, comment il les a référés à Dieu et comment ils ont soutenu sa charité jusqu'à son dernier soupir, il s'agit pour Gilberte de suggérer, autant qu'un mortel peut le pressentir, que son frère a reçu le don de persévérance, sans lequel nul ne peut être sauvé.

\section{Un esprit dérangé ?}

Si les hagiographes de Pascal tirèrent argument de sa maladie pour témoigner de sa sainteté, les sceptiques du siècle des Lumières en firent un usage diamétralement opposé : ils instrumentalisèrent ses souffrances afin de discréditer un penseur qui les embarrassait. Le port-royaliste, en tant qu'homme de science, leur apparaissait comme éminemment respectable: ses travaux avaient permis de ruiner la vieille science aristotélicienne, il avait défendu l'idée de progrès qui leur était chère, il fut partisan des modernes contre les anciens, et par surcroît pourfendeur des institutions sclérosées et

\footnotetext{
30 Ibid., p. 614.

31 Ibid., p. 641.
} 
des jésuites. Mais que s'avisa-t-il de verser dans le jansénisme le plus austère ? Le cilice, les attaques forcenées et dignes d'un «fanatique » contre le père Saint-Ange qu'il aurait vu brûler d'un bon ail pour un obscur point de théologie, son mode de vie gouverné par le plus incompréhensible masochisme, et surtout ce fatras antihumaniste des Pensées leur semblaient non seulement impardonnable, mais dangereux du fait même du talent de l'auteur, bien propre à persuader les âmes faibles. Aussi les philosophes éclairés mirent-ils tout en œuvre afin de distinguer deux Pascal : l'un, le scientifique et l'auteur des Petites Lettres, serait un authentique précurseur de l'esprit des Lumières ; l'autre, obscurantiste, confit en dévotions et rétrograde, serait un «fanatique » de la pire espèce, apôtre de «l'infâme ». Pour établir cette distinction, ils s'appuyèrent en particulier sur deux rumeurs mal vérifiées, qu'ils combinèrent de façon à répandre la légende noire d'un Pascal déséquilibré.

La première clabauderie concerne l'accident de carrosse, dont la véracité est mal attestée ; on la trouve dans un des Recueils Guerrier, où elle est présentée comme issue d'un manuscrit anonyme de l'Oratoire de Clermont :

M. Arnoul de Saint-Victor, curé de Chambourcy, dit qu'il a appris de M. le prieur de Barillon, ami de madame Perier, que M. Pascal, quelques années avant sa mort, étant allé, selon sa coutume, un jour de fête, à la promenade au pont de Neuilly avec quelques-uns de ses amis dans un carrosse à quatre ou six chevaux, les deux chevaux de volée prirent le frein aux dents à l'endroit du pont où il n'y avait point de garde-fou, et, s'étant précipités dans l'eau, les laisses qui les attachaient au train de derrière se rompirent, en sorte que le carrosse demeura sur le bord du précipice, ce qui fit prendre la résolution à $\mathrm{M}$. Pascal de rompre ses promenades et de vivre dans une entière solitude. ${ }^{32}$

Il est difficile de tirer argument d'un tel texte : le témoignage, qui n'est pas signé, relate un épisode inconnu jusque-là de la vie de Pascal, rapporté en outre de troisième ou quatrième main. On notera aussi que l'accident n'est pas précisément daté («quelques années avant sa mort»), et on ne sait par ailleurs rien d'une interruption par Pascal de ses promenades : on le voit au contraire, à la fin de sa vie, prendre en charge une jeune fille abandonnée rencontrée dans la rue et visiter les églises en guise de « divertissement ${ }^{33} »$. Il est possible que Pascal ait pu être pris dans une mésaventure qui se serait passée sur le pont de Neuilly, construit en bois et peu sécurisé : il serait

\footnotetext{
32 Ibid., p. 885 .

33 Ibid., p. 636.
} 
présomptueux d'en dire davantage. Le traumatisme ne dut pas avoir sur lui un grand retentissement, puisque, quelques mois avant sa mort, Pascal, avec quelques-uns de ses amis et en particulier le duc de Roannez, fut l'initiateur du premier système de transport en commun moderne de Paris, les « carrosses à cinq sols».

La seconde rumeur, plus ou moins avérée, concerne le gouffre que Pascal croyait voir s'ouvrir à son côté gauche. Elle est relatée par un proche de Port-Royal, l'abbé Boileau, qui s'emploie, dans une lettre de direction publiée après sa mort, à rassurer une demoiselle atteinte de terreurs imaginaires :

Cela me fait souvenir de M. Pascal dont la comparaison ne vous déplaira pas... Ce grand esprit croyait toujours voir un abîme à son côté gauche et y faisait mettre une chaise pour se rassurer; je sais l'histoire d'origine. Ses amis, son confesseur, son directeur avaient beau lui dire qu'il n'avait rien à craindre, il convenait de tout cela avec eux, car il n'était nullement visionnaire ; et un quart d'heure après, il se creusait de nouveau le précipice qui l'effrayait. ${ }^{34}$

Sans Voltaire et quelques jésuites mal intentionnés, cette lettre serait sans doute restée obscure et eût été rapidement oubliée. Mais l'auteur des Lettres anglaises s'en empara, et lui donna une publicité telle que l'anecdote eut un retentissement immense et vola de bouche en bouche :

Pascal croyait toujours voir un abîme à côté de sa chaise : faudrait-il pour cela que nous en imaginassions autant? [...] Vous trouverez dans les Mélanges de Leibniz que la mélancolie égara sur la fin la raison de Pascal ; il le dit même un peu durement. Il n'est pas étonnant, après tout, qu'un homme d'un tempérament délicat, d'une imagination triste, comme Pascal, soit, à force de mauvais régime, parvenu à déranger les organes de son cerveau. ${ }^{35}$

Rapidement, le courant rationaliste fit main basse sur ces anecdotes incertaines, non datées et contradictoires ; il les mêla avec des documents plus avérés, et les orchestra en suggérant qu'il existait un lien entre elles : l'accident aurait déréglé le système sensoriel de Blaise, et expliquerait le dérangement de ses facultés intellectuelles. Un Recueil de 1740, qui tente d'établir une connexion entre l'événement du pont et la conversion, fera l'objet d'une large exploitation par les adversaires de Port-Royal :

34 Lettres sur divers sujets de morale et de piété, Paris, 1737. Reproduit par J. Mesnard in Pascal, Euvres complètes, op. cit., t. I, p. 969.

35 Cité par Sainte-Beuve, Port-Royal, éd. Maxime Leroy, Gallimard, «Bibliothèque de la Pléiade », 1951-1953, t. II, p. 329. 
Il paraît bien que le Seigneur le poursuivait depuis longtemps, comme il l'avoua lui-même dans la suite. La Providence disposa divers événements pour le détacher peu à peu de ce qui était l'objet de ses passions. Un jour de fête, étant allé selon sa coutume, promener dans un carrosse à quatre ou six chevaux (au pont de Neuilly), les deux premiers prirent le mors aux dents à un endroit du pont où il n'y avait pas de garde-fous, et se précipitèrent dans la rivière. Comme les rênes se rompirent, le carrosse demeura sur le bord. Cet accident fit prendre à $\mathrm{M}$. Pascal la résolution de rompre ces promenades et de mener une vie plus retirée. Mais il était nécessaire que Dieu lui ôtât ce vain amour des sciences, auquel il était revenu; et ce fut pour cela, sans doute, qu'il lui fit avoir une vision, dont il n'a jamais parlé à personne, si ce n'est peut-être à son confesseur. On n'en a eu connaissance qu'après sa mort par un petit écrit de sa main qui fut trouvé sur lui. ${ }^{36}$

Les hommes des Lumières, qui ne se réfèrent jamais à la source originale, reprirent volontiers cet extrait, et adoptèrent la vision réductionniste de la conversion qui se trouve ici proposée : Pascal, terrifié d'avoir frôlé la mort, aurait aussitôt renoncé au monde et cherché dans la religion un moyen de se préserver de la crainte du trépas ${ }^{37}$. Non contents de prendre à leur compte cette interprétation sujette à caution, les philosophes créèrent de toutes pièces un lien entre l'accident et les étranges vertiges dont Pascal est supposé avoir été victime : l'épisode du pont de Neuilly est donné comme cause d'un désordre mental qui se traduisit non seulement par l'hallucination de la nuit de feu, mais aussi par la hantise du «gouffre » rapportée par l'abbé Boileau, et introduite ici sans raison dans le fil biographique. Pour donner de la crédibilité à leur montage, les philosophes datèrent en effet audacieusement de 1654 l'incident de circulation, et insinuèrent que celui-ci avait laissé dans l'esprit de la victime des «terreurs involontaires » dont la peur de l'abîme apparaît comme la manifestation la plus spectaculaire. Condorcet écrit ainsi, dans son prétendu Éloge de Pascal :

Ces recherches sur les fluides furent les derniers efforts de ce génie à qui la nature n'avait refusé que des organes proportionnés à sa force : ramené sans cesse à lui-même par la douleur, l'étude de l'homme fut la seule à laquelle son esprit, absorbé par la mélancolie, put alors se livrer. Cette mélancolie avait encore été augmentée par un accident singulier. Pascal était allé se promener à quatre chevaux et sans postillon, comme c'était alors l'usage. En passant sur le pont de Neuilly, qui n'avait pas de garde-fous, les deux premiers chevaux se précipitèrent. Déjà ils entraînaient la voiture dans la Seine ; mais heureusement les traits rompirent, et Pascal fut sauvé. Son imagination, qui conservait

36 Recueil de plusieurs pièces pour servir à l'histoire de Port-Royal [habituellement appelé Recueil d'Utrecht], Utrecht, 1740, p. 258. Le « petit écrit » ne peut être que le Mémorial.

37 Dinah Ribard a montré comment circulèrent ces « vignettes célèbres » : « elles sont très connues, mais personne ne sais plus au juste d'où elles viennent. Elles semblent en fait n'exister que pour garantir par la narration l'image stéréotypée qui reste d'un philosophe » (Raconter, vivre, penser: histoire(s) de philosophes, 1650-1766, Paris, Vrin/EHESS, « Contextes », 2003, p. 141). 
fortement les impressions qu'elle avait une fois reçues, fut troublée le reste de sa vie par des terreurs involontaires. On dit que souvent il croyait voir un précipice ouvert à côté de lui. Pascal ne pouvant ni chercher des ressources dans les sciences, ni trouver de repos en lui-même, n'eut plus d'espoir qu'en la religion. Jamais il n'avait cessé de l'aimer ; et elle fut, dans ses infirmités, sa consolation et son appui. ${ }^{38}$

Les expériences scientifiques et l'accident auraient ainsi débouché sur une psychose que seule la religion put alléger. Les philosophes mêlèrent ainsi adroitement vérité et mensonge, allégations sans fondement et témoignages douteux, de façon à construire un portrait à la fois saisissant et plausible de l'auteur des Pensées : l'inventeur du vide, hypersensible, terrorisé par la portée de ses propres découvertes, soumis à des traumas qui achevèrent de le déstabiliser, s'imaginait voir s'ouvrir un «vide » à côté de lui. C'était le génie qui succombait sous le poids trop lourd de ses propres productions, et sous le coup d'une nervosité à fleur de peau ${ }^{39}$ : il ne lui restait, pour échapper à la folie qu'on enferme, qu'à chercher secours dans les pratiques d'une religion morbide et destructrice, le «jansénisme ».

Cette habile machination permit aux hommes des Lumières de distinguer deux versants dans la pensée pascalienne : lorsqu'il traite des sciences, Blaise est sain d'esprit et son génie ne fait pas de doute; mais on peut récuser sans trop de scrupules la partie théologique de son œuvre, fruit du dérèglement de ses sens consécutif à l'accident ${ }^{40}$ :

Il faut pourtant oser le dire : quoiqu'en général le tableau que Pascal a fait de l'homme soit aussi vrai qu'il est fortement tracé, cependant dans ces pensées jetées au hasard, et que Pascal devait revoir, il lui en est échappé beaucoup de fausses. ${ }^{41}$

Une grande partie des Pensées serait ainsi marquée au sceau de la déraison, voire de la démence dans ce qu'elle a de plus inquiétant : après son renoncement à la physique et aux mathématiques, Pascal serait devenu un dangereux visionnaire. Condorcet tira les

38 Éloge et Pensées de Pascal, édition établie par Condorcet, annotée par Voltaire, édition critique par Richard Parish, in Les Euvres complètes de Voltaire, vol. 80A, Oxford, Voltaire Foundation, 2008, p. 90.

39 «L'accident de Neuilly permettait d'assujettir le génie de Pascal à son pathos », écrit ainsi Huguette Krief ( «D’un préservatif contre le fanatisme: la réédition par Voltaire de l'Éloge et Pensées de Pascal par Condorcet», Revue Voltaire, $\mathrm{n}^{\circ} 4$, 2004, p. 97). La critique analyse avec justesse cet amalgame : « L'argument qui consiste à relier l'enthousiasme de Pascal à un choc traumatique et à sa peur métaphysique du vide pour justifier ses choix théologiques est plausible sur le plan biographique », p. 108.

40 Antony McKenna écrit ainsi : «Voltaire avait distingué entre l'auteur génial des Lettres provinciales et le misanthrope malade des Pensées », Entre Descartes et Gassendi. La première édition des Pensées de Pascal, Paris, Universitas et Oxford, Voltaire Foundation, 1993, p. 12.

41 Éloge et Pensées de Pascal, op. cit., p. 118-119. 
conséquences de cette interprétation dans son édition des fragments pascaliens, reléguant en fin de volume les pensées relevant selon lui d'une incurable hypocondrie.

L'accident aurait ruiné les facultés d'un génie fragile, et précipité dans les visions auxquelles seul un pari irrationnel sur la foi pouvait porter secours : Voltaire fut ravi de ce séduisant distinguo qui permettait de sauver le savant et de condamner le janséniste. «Ce Blaise touchait donc à la fois à l'extrême force d'esprit et à l'extrême folie ? Cela est bon à savoir, on en peut tirer d'étranges conclusions », écrit à Condorcet le patriarche de Ferney ${ }^{42}$. Pour celui-ci, l'explication est simple, et gagne à être connue et répandue: «Qu'on ne se lasse pas de répéter que, depuis l'accident de Neuilly, le cerveau de Pascal était dérangé ». L'auteur des Pensées ne pouvait être, selon lui, qu'un «misanthrope sublime», travaillé par une sombre «mélancolie ${ }^{43} »$. C'est que, pour l'auteur des Lettres philosophiques, Pascal est le grand rival, le «géant », l'homme à abattre coûte que coûte : tout à l'efficacité de la polémique, Voltaire préfère les attaques ad hominem à une vraie discussion de fond.

De telles insinuations sur la santé psychiatrique de l'auteur des Pensées sont injustes, mais elles étaient si séduisantes aux yeux d'un lectorat qui ne demandait qu'à être convaincu, qu'on les retrouve encore très tard. Le courant scientiste qui domine la fin du XIX ${ }^{\mathrm{e}}$ siècle se contente ainsi de mettre au goût du jour l'argumentaire élaboré par les Lumières, en lui donnant une couleur médicale plus prononcée qui lui confère un air de scientificité. Dans un essai intitulé Génie et folie : réfutation d'un paradoxe, paru en 1898 dans les Annales médico-physiologiques, le Dr Albert Regnard s'emploie ainsi à montrer que génie et folie ne font jamais bon ménage, et que les grands hommes ont tous été parfaitement sains d'esprit. Pascal, sans faire exception, représente à ses yeux un argument a contrario : Regnard considère que Blaise a effectivement souffert de troubles mentaux, mais refuse toute valeur au fatras résultant du déséquilibre de son esprit.

42 Lettre à Condorcet, 7 septembre 1776. Voir Condorcet, Arithmétique politique : Textes rares ou inédits (17671789), édition critique commentée par Bernard Bru et Pierre Crépel, Paris, Institut national d'études démographique, PUF, 1994.

$43 \mathrm{XXV}^{\mathrm{e}}$ Lettre philosophique. 
On sait que cet accident de Neuilly fut le point de départ d'une hallucination souvent renouvelée, qui laissait entrevoir à Pascal l'horreur d'un abîme ouvert à ses pieds ; de plus, on trouve ici la trace d'une autre hallucination, d'une «vision» - pour parler comme les théologiens, - dont la réalité ne paraît pas moins évidente. ${ }^{44}$

«On sait que »: l'amalgame imaginé par les philosophes a décidément la vie dure. L'auteur ne cite les réserves de Sainte-Beuve face au texte de l'abbé Boileau que pour les rejeter d'un revers de la main : «il n'y a pas lieu vraiment de mettre en doute la véracité et l'exactitude de cet abbé ». Quant au Mémorial, qualifié comme chez Condorcet «d'amulette ${ }^{45}$ », le médecin considère qu'il « ressemble de tous points à ceux que les aliénés, dans les asiles, remettent journellement aux personnes qui les visitent ». Bien convaincu par une légende solidement établie, Regnard reprend sans la discuter la chronologie diffusée par Condorcet et Voltaire :

Quoi qu'il en soit, c'est à cette époque (13 novembre 1654), un mois environ après l'accident du pont de Neuilly, que s'accentue ce que Lélut appelle 'le retour à Dieu et à la maladie'. Pascal se retire du monde et ne cherche plus qu'à s'abêtir de son mieux. [Après cette date,] il ne travaille plus. ${ }^{46}$

L'itinéraire de Pascal confirme donc ainsi, aux yeux du médecin, sa thèse d'une incompatibilité du génie et de la maladie : Blaise donne l'exemple d'un talent supérieur gâché par des troubles mentaux qu'avait aggravé encore un contexte familial exalté par la religiosité janséniste :

Ce fut là, véritablement, un cas de banqueroute - non de la science, ce qui est une absurdité - mais du savant, du génie terrassé par la folie. Loin donc que celle-ci soit une condition de celui-là, - quand, par malheur, ils viennent à coexister, entre les deux la lutte est à mort, et c'est toujours le génie qui succombe..$^{47}$

Quelque peu convaincante et même spécieuse que fut, au plan scientifique, la construction échafaudée par les adversaires de Port-Royal, elle fut jugée si plausible qu'elle fit longtemps autorité auprès des esprits forts.

\section{L'homme de génie et la mélancolie : le Pascal des Romantiques}

44 Génie et folie, p. 31.

45 L'expression «d'amulette mystique », qui connut un certain succès avant que Bruncschvicg n'impose le terme de Mémorial, avait été inventée par Condorcet dans son édition des Pensées (Londres, 1776, p 504).

46 Ibid., p. 36-37.

47 Ibid., p. 42. 
Par un retournement à cent quatre-vingts degrés dont l'histoire littéraire possède seule le secret, les Romantiques s'employèrent à réhabiliter Pascal malmené par les Lumières: ils ne lurent pas les Pensées comme la production d'un fanatique, mais comme l'œuvre d'un esprit dont la puissance est hors du commun. On connait la description fameuse laissée par Chateaubriand :

Il y avait un homme qui à douze ans avec des barres et des ronds, avait créé les mathématiques; qui à seize avait fait le plus savant traité des coniques qu'on eût vu depuis l'antiquité ; qui à dix-neuf réduisit en machine une science qui existe tout entière dans l'entendement; qui à vingt-trois ans démontra les phénomènes de la pesanteur de l'air, et détruisit une des grandes erreurs de l'ancienne physique ; qui à cet âge où les autres hommes commencent à peine de naître, ayant achevé de parcourir le cercle des sciences humaines, s'aperçut de leur néant, et tourna ses pensées vers la religion; qui depuis ce moment jusqu'à sa mort, arrivée dans sa trente-neuvième année, toujours infirme et souffrant, fixa la langue que parlèrent Bossuet et Racine, donna le modèle de la plus parfaite plaisanterie comme du raisonnement le plus fort ; enfin, qui, dans les courts intervalles de ses maux, résolut par abstraction un des plus hauts problèmes de géométrie et jeta sur le papier des pensées qui tiennent autant du dieu que de l'homme : cet effrayant génie se nommait Blaise Pascal. ${ }^{48}$

Indignés par les propos haineux tenus par les hommes des Lumières, les Romantiques ne revinrent pas pour autant sur le diagnostic morbide des philosophes : pour eux aussi, Pascal est un malade - mais pour eux cette maladie est le vrai secret de son génie ${ }^{49}$. Leur conception de la création s'accommodait fort bien en effet d'un tempérament nerveux. Retournant contre eux le discours des rationalistes, qui utilisaient la folie prétendue de l'auteur des Pensées pour retrancher de son œuvre ce qui leur déplaisait, et l'amputer en particulier de sa dimension religieuse et mystique, ils mirent tout leur effort à montrer que Pascal, sublimant ses maux pour en faire de l'art, métamorphosant la boue pour en tirer de l'or, fut un héros romantique avant la lettre. Reprenant le propos de Voltaire, Chateaubriand ne nie pas que l'auteur des Pensées ait été un «fou sublime » (sic), mais suggère que cette folie, ce fut celle de la croix, et qu'elle lui permit d'atteindre des hauteurs de pensée auxquelles il n'eût pas pu accéder s'il n'eût été chrétien. Pascal, selon Chateaubriand, est ainsi un «grand modèle de mélancolie ${ }^{50}$ ». Le mot est loin d'être neutre: les Romantiques, en exacerbant l'idée d'une liaison entre génie et

\footnotetext{
48 F.R. de Chateaubriand, Génie du Christianisme, [1802], partie III, livre II, chapitre 6, « Suite des moralistes ».

49 J. J. Demorest, « Pascal et les premiers Romantiques », French Review, mai 1949, p. 436.

50 Chateaubriand cité par Desmorest, art. cit., p. 441.
} 
disposition morbide, réactivent le stéréotype du génie mélancolique, culturellement surdéterminé depuis le Problème $X X X^{51}$.

Baudelaire, au terme du retour en grâce de Pascal entamé quelques décennies plus tôt, brosse un saisissant portrait de l'auteur des Pensées : reprenant pour les détourner les stéréotypes calomnieux répandus par les Lumières, le poète, qui voit dans le portroyaliste son double, nous le présente comme saisi par une vertigineuse angoisse que provoque l'hallucination de «l'abîme »:

\section{Le Gouffre}

Pascal avait son gouffre, avec lui se mouvant.

- Hélas! tout est abîme, - action, désir, rêve,

Parole ! et sur mon poil qui tout droit se relève

Maintes fois de la Peur je sens passer le vent.

En haut, en bas, partout, la profondeur, la grève,

Le silence, l'espace affreux et captivant...

Sur le fond de mes nuits Dieu de son doigt savant

Dessine un cauchemar multiforme et sans trêve.

J'ai peur du sommeil comme on a peur d'un grand trou,

Tout plein de vague horreur, menant on ne sait où ;

Je ne vois qu'infini par toutes les fenêtres,

Et mon esprit, toujours du vertige hanté,

Jalouse du néant l'insensibilité.

Ah ! ne jamais sortir des Nombres et des Êtres !52

On reconnaît dans ce poème bien des échos des Pensées; les vers évoquent plusieurs des grands motifs de l'imaginaire pascalien : l' «abîme », le «vertige », le «gouffre » renvoient à une agoraphobie provoquée par les «espaces » infinis au milieu desquels l'homme se trouve plongé, décentré et perdu dans un milieu indéfini, « en haut, en bas, partout ». Mais malgré cette reprise fidèle des thèmes pascaliens, la conception du monde et de l'homme qui sous-tend ces vers est profondément différente de celle du port-royaliste : Baudelaire transforme l'apologiste en un être saisi d'angoisse. Le Pascal de Baudelaire est un être tragique, terrifié non seulement par le gouffre, mais aussi par les espaces infinis, et même par le divin : «Dieu... dessine un cauchemar ». Hanté par

\footnotetext{
51 Op. cit.

52 Charles Baudelaire, Les Fleurs du mal, Introduction de Claude Pichois, Poésie/Gallimard, 1972, p. 220.
} 
le désir de mort, il «jalouse du néant l'insensibilité »; quelques déplacements suffisent pour transformer profondément la tonalité d'un même thème : les « espaces infinis » deviennent chez Baudelaire à la fois «affreux et captivants », en un magnifique oxymore que ne se serait pas permis l'apologiste, car chez lui, l'univers sans limites ne provoque pas l'obscur désir de s'y perdre. Cette «peur », à laquelle Baudelaire ne voit comme antidote que la perte de conscience, ce ne peut être celle de Pascal pour qui au contraire «pensée fait la grandeur de l'homme»; le fragment du roseau pensant explique que l'être humain est supérieur à l'univers qui le broie précisément grâce à sa conscience indestructible. Baudelaire, tourmenté par l'angoisse, croit voir dans Pascal son frère, mais cette fraternité rêvée repose sur un malentendu ${ }^{53}$.

Si la veine rationaliste étend ses ramifications jusqu'aux premières décennies du $\mathrm{XX}^{\mathrm{e}}$ siècle, l'imagerie romantique court jusqu'à nos jours; la corrélation entre tempérament maladif et émergence du génie, sans être nécessairement explicitée telle quelle, figure en effet au fronton des biographies pascaliennes récentes : du Génie de Pascal de Jean Guitton ${ }^{54}$ à Pascal ou le génie français de Jacques Attali ${ }^{55}$, c'est toujours le secret de l'excellence intellectuelle et créative de Pascal que les auteurs tentent de percer ; dans cette perspective, la ou les maladies qui affectèrent le jeune Auvergnat constituent bien souvent, à leurs yeux, une clef précieuse.

Au temps de la légende, qu'elle fût noire ou dorée, succéda celui de l'analyse minutieuse et scientifique. Sainte-Beuve, dans son ouvrage sur Port-Royal, s'interroge à son tour sur la prétendue maladie de Pascal: «N'aurions-nous point affaire tout simplement à un malade, à un visionnaire, je n'invente point les termes, à un halluciné ?

53 Voir, sur les relations complexes entre ces deux écrivains, Jean Dubray, Pascal et Baudelaire, Paris, Classiques Garnier, « Lire le XVII ${ }^{\mathrm{e}}$ siècle », 9, 2011.

54 Paris, Aubier, 1962. Guitton écrit dans le «Discours sur le génie de Pascal » qui ouvre le volume : « Entre 23 et 62 , dans cet instant de trente-neuf ans qui fut pour nous un intervalle de tant d'angoisses, - lui, sachant ce qu'il voulait faire, dominant, utilisant l'inquiétude, obéissant aux signes, a déployé un génie admirable » (p. 10). Le talent de Pascal procède, selon l'auteur, d'une maîtrise et pour ainsi dire d'une sublimation de la détresse qui le tenaillait.

55 Jacques Attali, Blaise Pascal ou le génie français, Paris, Fayard, 2000 : « À trente-cinq ans, souffrant le martyre, il résout l'un des plus difficiles problèmes de mathématiques jamais posé [...] follement épris d'une sœur sans doute aussi géniale et refoulée que lui » (p. 12 et 14). 
Pascal, en un mot, comme on l'a dit de Lucrèce, n'a-t-il pas eu sur la fin un véritable égarement de la raison ?». L'historien mène alors l'enquête. Il retrouve la source de l'anecdote sur le gouffre, et excelle à montrer qu'on ne peut tirer grand-chose de ce texte tardif : il note que le récit, non daté, n'est pas corroboré par les Vies de Pascal, qui nous présentent au contraire l'auteur des Pensées marchant dans la rue sans difficulté et sans abîme ; le Journal des Savants, dans sa recension des Lettres de l'abbé Boileau, précise de son côté à propos de cette historiette : «Nous n'en avions jamais entendu parler ». Et Sainte-Beuve de persifler :

Si un de ces savants qui se piquent de rigueur, si un physiologiste venait, sur la foi de cette anecdote, réclamer Pascal comme un de ses malades et faisait mine de le traiter en conséquence, oh! alors au nom du bon sens comme du bon goût, nous lui dirions: Holà ! 56

Après un bon siècle passé à insister sur l'état de santé déplorable de Pascal, et à justifier par là ses errances ou ses fulgurances, Sainte-Beuve tend à minimiser l'effet de la maladie sur la vie et, partant, l'œuvre de l'auteur des Pensées :

Sans prétendre nier les singuliers accidents nerveux de Pascal, et leur contrecoup sur son humeur ou sur sa pensée, nous maintenons qu'à cette distance, et dans l'état des renseignements transmis, il n'y a lieu à venir asseoir là-dessus aucun diagnostic, comme on dit. Ce qui me paraît au contraire positif, c'est que, si malade des nerfs qu'on le voie en effet, Pascal demeura jusqu'à la fin dans l'intégrité de sa conscience morale et de son entendement. Le reste nous échappe. ${ }^{57}$

Il n'est pas indifférent qu'une observation aussi prudente émane de la plume d'un critique qui fut, plus que tout autre, attentif à repérer, chez un auteur, l'origine biographique de son écriture.

Les successeurs sérieux de Sainte-Beuve feront preuve de la même réserve. Gonzague Truc, dans sa «biographie psychologique ${ }^{58} »$ de Pascal, prend soin d'avertir son lecteur au moment où il aborde la question de sa maladie, «dont il faut se garder d'interpréter indiscrètement les données ». S'appuyant sur les travaux de plusieurs médecins ${ }^{59}$, il attribue le «pittoresque » récit de la sorcière au carreau, et démystifie les

56 Port-Royal, op. cit., t. II, p. 332.

57 Ibid.

58 Gonzague Truc, Pascal, son temps et le nôtre, Paris, Éditions Albin Michel, 1949, «Biographie historique », p. 16.

59 En particulier sur un fascicule des Archives de philosophie rédigé par le Dr. Robert de Sinéty (1923, vol. I, cahier 
légendes concernant le carrosse du pont de Neuilly, le gouffre et les visions. Contrairement à Maurras, avec qui il était pourtant lié, Truc refuse de croire que Blaise ait été victime d'hallucinations : «Non, l'auteur des Provinciales, des Pensées, et même du 'mystère de Jésus' n'était pas fou ${ }^{60} »$. Au vu de l'incertitude des sources, il ajoute, à propos de la phobie parentale dont aurait souffert le petit Blaise : «le complexe d'Edipe chez Pascal, quelle aubaine ! Nous n'en tirerons rien ${ }^{61} \gg$.

On le voit, au terme de ce rapide parcours, l'examen d'une éventuelle sublimation ou conversion des souffrances en création a été si influencée par les différentes appropriations, ou récupérations, dont Pascal a fait l'objet, qu'il est difficile de l'évaluer avec la sérénité et l'objectivité nécessaires à cette analyse.

\section{Les Pensées : œuvre traumatique ${ }^{2}$}

À travers les siècles, les lecteurs se sont emparés, chacun à leur manière, de la maladie de Pascal, pour en tirer des conclusions divergentes, voire opposées. Est-ce à dire qu'il faille renoncer à traiter la question ? Il serait aussi vain que présomptueux de rechercher dans la biographie pascalienne l'étiologie d'un génie qui, assurément, ne saurait se laisser réduire à l'énumération de troubles corporels - ou mentaux. Reste, pour tenter d'éclairer la question depuis un angle différent, à considérer les textes euxmêmes.

Penchons-nous d'abord sur le Mémorial ${ }^{63}$, que Pascal gardait, cousu dans son vêtement, depuis son expérience mystique de novembre 1654. Peut-on y discerner la trace d'une épreuve sentie comme un traumatisme ? Les rationalistes n'hésitent pas à voir dans cet événement l'une de ces hallucinations résultant de l'accident de Neuilly. Condorcet parle de «folie » pour qualifier cet éblouissement mystique. Mais si l'on se

III).

60 Gonzague Truc, op. cit., p. 16.

61 Ibid.

62 Nous citerons les pensées d'après la numérotation établie par Philippe Sellier (Paris, Bordas, «Classiques Garnier », 1993).

63 Voir, sur ce texe, Hélène Michon, «L'écriture mystique du Mémorial », in Pascal, auteur spirituel, op. cit., p. 163-185 et Jean Mesnard, « Bible et liturgie dans le Mémorial », ibid., p. 187-217. 
fie au seul texte, la relation intime avec la divinité fut vécue sur le mode de la paix, de la joie et de la douceur, et en rien comme un foudroiement jupitérien.

Certitude, certitude, sentiment, joie, paix [...]

Joie, joie, joie, pleurs de joie [...]

Renonciation totale et douce $[\ldots]$ (fr. 742)

De telles expressions ne paraissent pas corroborer la thèse d'une blessure psychique. C'est toute la confiance d'un cœur épris de la tendresse de Dieu qui s'épanche dans ce poème incandescent, quoi qu'on puisse penser de l'origine et de la nature exacte de l'expérience mystique. Le «Mystère de Jésus », autre texte privé que Pascal n'aurait jamais songé à publier, fait entendre des échos semblables, rassurants. Le ton du dialogue est celui de la consolation, du réconfort, de l'apaisement : «Console-toi. Tu ne me chercherais pas si tu ne m'avais trouvé » (fr. 751). Lorsqu'on considère la Lettre sur la mort de son père, écrite à Florin Périer, son beau-frère, ce n'est pas l'expression du traumatisme causé par cette disparition qui frappe le lecteur, mais plutôt la sérénité de l'épistolier, qui s'attache à replacer ce trépas dans une perspective chrétienne, de façon à montrer qu'elle n'est «horrible » qu'aux yeux du siècle corrompu : certes, «sans Jésus-Christ, [la mort] est horrible, elle est détestable, et l'horreur de la nature ». Mais « en Jésus-Christ elle est tout autre : elle est aimable, sainte, et la joie du fidèle. Tout est doux en Jésus-Christ ». Pascal euphémise la mort en lui donnant son véritable sens : les « païens » voient « une charogne infecte, car la nature trompeuse le figure de la sorte », mais les chrétiens reconnaissent dans le trépassé «le temple inviolable et éternel du Saint-Esprit ${ }^{64} »$. Le paradoxe mérite qu'on s'y arrête : le fils aimant s'emploie, dans cette lettre, à consoler de sa douleur, en vrai chrétien, le mari de Gilberte, qui, bien que n'étant pas lié par le sang à Étienne, semble plus troublé que Blaise par cette disparition. La douleur de son beau-frère, sans doute légitime, paraît trop vive aux yeux du converti :

Ce n'est pas que je souhaite que vous soyez sans ressentiment : le coup est trop sensible, il serait même insupportable sans un secours surnaturel. Il n'est donc pas juste que nous soyons sans douleur comme des anges qui n'ont aucun sentiment de la nature; mais il n'est pas juste aussi que nous soyons sans consolation comme des païens qui n'ont aucun sentiment de la grâce ; mais il est juste que nous soyons affligés et consolés comme

64 «Lettre sur la mort de son père », in Euvres complètes, op. cit., II, p. 854-856. 
chrétiens, et que la consolation de la grâce l'emporte par-dessus les sentiments de la nature. ${ }^{65}$

Pascal le jeune possède le secours de la religion, qui l'empêche de désespérer et de vivre trop douloureusement cette mort. Les «accidents de la vie», pour reprendre son expression, ne doivent pas nous toucher exagérément, et doivent toujours être mesurés à l'aune du sacrifice du Christ, dont l'existence du chrétien doit être une imitation : aussi convient-il que ces «accidents de la vie ne fassent d'impression dans l'esprit des chrétiens qu'à proportion qu'ils interrompent ou qu'ils accomplissent ce sacrifice ${ }^{66} \gg$.

Quand on lit les fragments des Pensées destinés à trouver place dans le livre sur la religion que Pascal projetait, l'impression est très différente, et l'on pressent que, si les commentateurs ont accordé une si grande place aux troubles pascaliens, c'est que l'Apologie se prêtait particulièrement bien à cette approche. Les fragments évoquent souvent des situations angoissantes : des soldats à la guerre (fr. 84), un prisonnier dans un cachot (fr. 195), le dénouement sanglant d'une tragédie (fr. 197), un naufragé égaré sur une île déserte (fr. 229), voire un astronaute avant la lettre perdu dans l'espace infini (fr. 233), autant d'états menaçants et terrifiants que Pascal imagine comme à plaisir. Le décor des Pensées, minutieusement étudié par Philippe Sellier dont nous reprenons ici les conclusions ${ }^{67}$, est lui aussi sinistre et propre à susciter des alarmes: océans nocturnes, fleuves de feu (fr. 460) ou marécages fangeux constituent le cadre inquiétant des Pensées: «l'homme n'est qu'un roseau »(fr. 231). Ces marais fétides entraînent tout naturellement un imaginaire paludéen : comment ces tourbières malsaines ne provoqueraient-elles pas toutes sortes d'infections ? Comment cette atmosphère de pestilence ne déboucherait-elle pas sur la description d'états morbides ? Ces images de maladie, de corruption, de pourriture et de putréfaction servent à Pascal à décrire le mal mortel qui ronge l'être humain, et qui n'est autre que le péché : «Que le cœur de l'homme est creux et plein d'ordure!» (fr. 171). Le cœur est une citerne suintante (« ...la citerne d'où vous êtes tirés », fr. 718); l'homme est un «cloaque d'incertitude

65 Ibid., p. 860.

66 Ibid., p. 853-854.

67 Voir en particulier, Pascal et saint Augustin, op. cit., «Fluidité nocturne du monde », p. 20-36, ainsi que divers articles réunis dans Port-Royal et la littérature. Pascal, op. cit., en particulier « 'Sur les fleuves de Babylone' : la fluidité du monde et la recherche de la permanence », p. 411-423. 
et d'erreur » (fr. 164), un «ver de terre » (fr. 164) rampant dans la vase : on voit se dessiner un paysage d'eaux impures qui constitue aussi bien le monde où nous vivons que notre propre cœur sans fond. Comme Job, l'homme est un malade, plein « d'abcès », en proie à des «fièvres » (fr. 587), et incurable sans la grâce. Ces marais où nous traînons sans espoir sont d'autant plus inquiétants que Pascal se les représente plongés dans la nuit : roseaux pensants, nous passons notre vie près de fleuves en feu, et dans une obscurité silencieuse. Pour punition de nos crimes, nous errons dans des bourbiers nocturnes, «égarés, avec inquiétude et sans succès, dans des ténèbres impénétrables »(fr. 19), perdus sans espoir de trouver le chemin. Paraphrasant Isaïe, Pascal écrit :

Nous avons attendu la lumière et nous ne trouvons que les ténèbres. Nous avons espéré la clarté et nous marchons dans l'obscurité. Nous avons tâté contre la muraille comme des aveugles, nous avons heurté en plein midi, comme au milieu d'une nuit, et comme des morts en des lieux ténébreux. (fr. 73568)

Sans lumière ni pilote, l'homme est abandonné dans ce paysage désolé : «l'homme [est] sans lumière abandonné à lui-même et comme égaré » (fr. 229). Il ne lui reste qu'à tenter de voguer sur ces marais, au milieu de fleuves embrasés, sans même un falot pour le guider : « Nous voguons sur un milieu vaste, toujours incertains et flottants, poussés d'un bout vers l'autre » (fr. 230). L'image dont se sert Pascal pour figurer cet homme impuissant et livré à lui-même n'est pas celle du labyrinthe, mais celle du grand espace vide et silencieux : «l'univers » est «muet» (fr. 229). Ce cosmos démesuré est d'autant plus terrifiant que vient s'y greffer une peur panique de la chute, en un vertige incontrôlable. L'homme est réellement «tombé de son vrai lieu » (fr. 19), les péchés nous entraînent au fond d'un gouffre : « nous courons sans souci dans le précipice » (fr. 198), et c'est encore un abîme que Pascal ouvre sous les pieds du «plus grand philosophe du monde », dans le fameux fragment sur l'imagination (fr. 78). C'est ainsi l'angoisse des espaces vides doublée d'une crainte de tomber, principaux constituants du syndrome d'agoraphobie, qui caractérise le rapport à l'espace dans les Pensées: « notre raison est toujours déçue par l'inconstance des apparences : rien ne peut fixer le fini entre les deux infinis qui l'enferment et le fuient» (fr. 230). Mais, dans un 
mouvement de renversement surprenant, l'espace indéfini et sans limites rassurantes devient un «cachot» où l'homme est enfermé (fr. 230), comme écrasé par le poids de cet univers qui l'accable; d'où les termes récurrents d'enfermement et la métaphore de la prison, qui reviennent souvent dans les Pensées: l'agoraphobie débouche ainsi paradoxalement et simultanément sur une impression de claustrophobie ${ }^{69}$.

Les pensées offrent ainsi une constellation de scénarios fortement anxiogènes, de décors sinistres et de figures épouvantables : l'abîme, à défaut de s'ouvrir sur son côté gauche, est omniprésent dans l'œeuvre de Pascal. Est-ce à dire que celui-ci jetait sur le papier, pour les exorciser, ses propres terreurs ? Ce n'est pas sûr, et on peut même proposer l'hypothèse inverse. La situation d'énonciation dans laquelle se trouve Pascal, obligé de convaincre un athée insouciant ou hostile, implique la nécessité de « remuer » et de «travailler » ce libertin; pour lui faire quitter son indifférence métaphysique et lui faire prendre conscience de la misère de sa condition, il doit d'abord l'inquiéter, le sortir de la confortable tranquillité où il passe ses jours, avant de proposer la religion chrétienne comme consolation à l'angoisse qu'il a préalablement provoquée. On sait maintenant, grâce aux travaux de Jean Mesnard ${ }^{70}$, que le fragment des espaces infinis (fr. 232) n'exprime pas, comme le croyait Baudelaire, l'angoisse éprouvée par Pascal au regard de l'immensité insondable de l'espace galiléen ; au contraire, Pascal, chrétien éclairé par la grâce, n'est pas sujet à ce vertige des infinis, car il sait, au-delà des apparences, percevoir le doigt de Dieu dans le monde (fr. 38). La célèbre phrase a plutôt pour objet de creuser un abîme devant les libertins, et de leur faire éprouver une blessure narcissique, conséquence de la faillite de leur entendement incapable de comprendre les deux infinis qui broient l'être humain. Pascal n'ouvre devant le libertin l'immensité vertigineuse de l'univers que pour lui faire expérimenter la défaite sans rémission de sa raison.

Une rhétorique de l'émotion et de l'inquiétude, destinée à mettre en scène ces traumas, domine l'apologie. Dieu étant «sensible au cœur », il convient, en usant d'une

69 Sur la thématique des Pensées, voir les travaux de Philippe Sellier et en particulier son Pascal et saint Augustin, op. cit., ainsi que, du même auteur, la préface de l'édition citée des Pensées, p. 39-52, «L'univers des Pensées ».

70 Jean Mesnard, «Le cri célèbre: 'Le silence éternel de ces espaces infinis m'effraie' doit être placé dans la bouche de l'incrédule », p. 167. 
éloquence particulière, d'amollir le cœur du libertin pour lui faire quitter les froides et faibles «certitudes » où il se complaît. Pour Pascal, l'écriture est une lutte : «Rien ne nous plaît que le combat» (fr. 637) ; écrire une apologie, c'est harceler sans cesse le lecteur comme une armée harcèle l'ennemi : «je ne souffrirai point qu'il repose » (fr. 703). Pour offusquer son destinataire, le théologien emploie d'audacieuses techniques qui doivent plus à Démosthène qu'à Cicéron et procèdent, selon Marc Fumaroli, d'un authentique sublime longinien ${ }^{71}$. L'écriture est conçue pour frapper l'imagination et ainsi «s'insinuer» dans l'esprit du lecteur, fût-ce à son corps défendant, au point que le libertin des Pensées se surprendra, malgré lui, à citer Pascal :

La manière d'écrire d'Épictète, de Montaigne et de Salomon de Tultie est la plus d'usage, qui s'insinue le mieux, qui demeure le plus dans la mémoire, et qui se fait le plus citer. (fr. 618)

Les mots sont, selon le mot de Philippe Sellier, des «coups de fouet», voire des marques au fer rouge, dont le libertin ne se remettra jamais. Du traumatisme, on passe en quelque sorte à un conditionnement subliminal. La violence épigrammatique (fr. 650), les tournures archaïques (fr. 77 ; 678 ; 94), l'effet de chute brutale (fr. 550), les invectives véhémentes (fr. 164: «Connaissez donc, superbe...»), les impératifs («Taisez-vous, nature imbécile», fr. 164): telles sont quelques-unes des armes polémiques de l'arsenal pascalien. Arsenal redoutablement efficace : plusieurs de ces maximes sont passées en proverbes ${ }^{72}$.

\section{Conclusion}

Ainsi, quels que puissent être les éléments qui, dans le bilan de santé de Pascal, invitent à une telle analyse, c'est le principe même d'une recherche portant sur une éventuelle causalité biologique du génie qui mérite d'être réévalué. En l'absence de sources suffisantes, il est presque impossible de mesurer quelle put être l'influence des troubles éprouvés par Pascal sur sa création. Il est en revanche aisément possible

71 Marc Fumaroli, «Pascal et la tradition rhétorique gallicane», in Méthodes chez Pascal. Actes du colloque de Clermont-Ferrand, Paris, P.U.F., 1979, p. 359-372.

72 Sur cet arsenal de la rhétorique pascalienne, voir Philippe Sellier, « Des figures originaires aux découvertes de 1656 », dans l'édition citée des Pensées, p. 67-74. 
d'attribuer la force traumatique des Pensées à une stratégie rhétorique destinée à perturber la sérénité de son lecteur libertin, afin de créer en lui une disponibilité à la grâce, pour le cas éventuel où Dieu aurait décidé de la lui accorder. Rationalistes et romantiques ont, pendant longtemps, pris à tort pour l'expression d'une angoisse personnelle des techniques qui relèvent d'une stratégie argumentative. Le contresens, qui inspira aussi bien les invectives voltairiennes que certains des vers les plus poignants de Baudelaire, a beau avoir été fécond, il n'en est pas moins un contresens. Reste l'œuvre elle-même: les pages les plus intimes ne laissent rien percer d'une éventuelle angoisse pascalienne ; elles résonnent plutôt comme un chant où dominent l'apaisement et la consolation. Seules les Pensées sont un dispositif conçu spécialement pour bouleverser le lecteur, le laisser pantelant, privé à tout jamais de sa tranquillité satisfaite. Si la notion de traumatisme prend ici tout son sens, c'est dans la mesure où Pascal inflige à son lecteur une «blessure narcissique » et lui fait découvrir, jusqu'à le saisir d'horreur, la dévastation provoquée par ce traumatisme originaire qu'est le péché - et dont il ne pourra être libéré que par l'infusion de la grâce. 